\title{
Estrategias para mejorar la calidad de vida laboral: la perspectiva de las enfermeras
}

Strategies to Improve Quality of Working Life: The Perspective of Nurses

\section{Alberto Granero-Lázaro ${ }^{1}$, Josep M Blanch-Ribas ${ }^{2}$, Juan Francisco Roldán-Merino ${ }^{3}$, Anna Maria Escayola-Maranges ${ }^{1}$}

${ }^{1}$ Centro de Salud Mental. Parc Taulí Hospital Universitario. Sabadell, España.

${ }^{2}$ Departamento de Psicología Social. Facultad de Psicología. Universidad Autónoma de Barcelona (UAB). Barcelona, España.

${ }^{3}$ Escuela Universitaria de Enfermería Sant Joan de Déu. Campus Docente Sant Joan de Déu. Universidad de Barcelona (UB). Barcelona, España.

Contacto: albertgranerolazaro@gmail.com

Fecha de recepción: 20 de mayo de 2017 / Fecha de aceptación: 15 de julio de 2017

\section{Resumen}

Objetivo principal: Explorar los aspectos que mejorarían la calidad de vida laboral (CVL) desde la visión enfermera.

Metodología: El diseño fue descriptivo y transversal. La muestra fue aleatoria y participaron 1760 enfermeras de la provincia de Barcelona. Se realizó un análisis cualitativo de contenido, de tipo exploratorio descriptivo, sobre los aspectos que mejorarían la CVL según las enfermeras.

Resultados principales: Las enfermeras opinaron que aumentaría su CVL si mejoran las condiciones de trabajo (CT) destacando entre otros aspectos el entorno, la prevención de riesgos, el respeto, el reconocimiento, la contratación, la conciliación, el tiempo de trabajo, la retribución, la carga y organización del trabajo, el ratio enfermera/paciente, la autonomía, la participación, el liderazgo, el desarrollo y la promoción profesional.

Conclusión principal: Las expectativas de mejora de la CVL son individuales, están asociadas a la mejora de las CT y relacionadas con las condiciones laborales, profesionales y personales de cada enfermera.

Palabras clave: enfermeras, condiciones de trabajo, calidad de vida laboral, estrés psicológico, agotamiento profesional, satisfacción laboral, insatisfacción laboral, bienestar laboral, malestar laboral.

\begin{abstract}
Objective: To explore the nurses' perspective of the aspects that would improve the quality of working life (QWL).

Methods: It was a descriptive and transversal design. The sample was randomized and 1760 nurses participated all over the province of Barcelona. A qualitative, descriptive exploratory analysis of the content was done of the aspects that would enhance the QWL in the nurses' opinion.

Results: Nurses felt that would increase their QWL the improvement of working conditions (WC) highlighting the environment, prevention of occupational hazards, respect, recognition, recruitment, conciliation, schedules, pay, workload, work organization, the nurse/patient ratio, autonomy, participation, leadership, professional development and promotion.

Conclusions: The expectations of improving the QWL are individual, are associated to improve WC and related to labour, professional and personal conditions of each nurse.
\end{abstract}

Keywords: nurses, working conditions, quality of life, stress psychological, burnout professional, job satisfaction, job dissatisfaction, labour welfare, labour malaise.

\section{Introducción}

Las condiciones de trabajo (CT) donde se desarrolla la actividad y las relaciones laborales son determinantes para evaluar la calidad de vida laboral ${ }^{1}$ (CVL). En el pasado se habló más desde una perspectiva negativa, utilizando términos como insatisfacción, estrés y burnout, pero desde hace años se habla más de prevención de riesgos psicosociales, bienestar laboral y CVL. Este cambio está amparado por la Ley 31/1995 de 8 noviembre de Prevención de Riesgos Laborales ${ }^{2}$, que hizo que las organizaciones empezaran a realizar eva- 
luaciones de los riesgos psicosociales y a implementar intervenciones dirigidas a minimizarlos y a mejorar el bienestar laboral de los profesionales.

Sin embargo, el contexto socioeconómico y político de la última década no ha sido favorable para la implementación de estas intervenciones en el entorno sanitario. El incremento de los costes en salud, el aumento de las expectativas respecto a la sanidad, los avances tecnológicos, la crisis económica, los cambios en las políticas de financiación y de seguridad de los pacientes son factores que han determinado la necesidad de transformación de las organizaciones sanitarias ${ }^{3,4}$, para ser más ágiles y rentables, contener el gasto, contratar y retener profesionales excelentes, dar respuesta a las necesidades de los pacientes, mejorar la seguridad y la calidad asistencial y ser más eficientes. Algunos de éstos cambios han empeorado las $\mathrm{CT}$, que comportan altos niveles de atención y concentración, elevada responsabilidad, sobrecarga y largos y desordenados horarios y turnos, situaciones que han contribuido a que los riesgos psicosociales en el entorno laboral estén más presentes ${ }^{5,6,7}$.

El Instituto Nacional de Seguridad e Higiene en el Trabajo (INSHT) ${ }^{8}$ y autores como Lavoie ${ }^{5}$, Jourdain", Cortese $^{10}$, Appelbaum $^{11}$, Riahi ${ }^{12}$, Arteman ${ }^{13}$ o Purcell ${ }^{14}$ han considerado entre los factores de riesgo psicosocial las condiciones ambientales (iluminación, ruido, temperatura, etc.) y los factores relacionados con la organización y la gestión del trabajo (descansos, horario, turnos, tareas, ritmo, monotonía, autonomía, formación, responsabilidad, desempeño del rol, comunicación, estilo de mando, participación en las decisiones, soporte de los gestores, competencia, carga mental y física, relaciones interpersonales, condiciones de empleo y desarrollo profesional). La coexistencia y persistencia de determinados factores de riesgo pueden sobrepasar la capacidad de afrontamiento de la persona generándole situaciones de malestar, estrés, insatisfacción, burnout o alteraciones de la salud física y mental $^{6,13,15}$ que ponen en peligro el sentido del trabajo, el bienestar laboral y la CVL.

Estudios como el de Villalobos ${ }^{16}$ han evidenciado la relación entre los riesgos psicosociales y el incremento de procesos mórbidos en los trabajadores, las bajas y el absentismo laboral $^{17}$. Letvak ${ }^{18}$ y Aiken ${ }^{3,15}$, han relacionado la salud de los profesionales con la calidad del cuidado, observando que los profesionales con problemas de salud tienen menor rendimiento, provocan cansancio en los compañeros y dan una atención de peor calidad. El nivel de satisfacción profesional y el entorno laboral también se han relacionado con la calidad de los servicios sanitarios ${ }^{15}$, evidenciándose que los profesionales con satisfacción alta dan un servicio de mayor calidad y son más resolutivos frente a las necesidades de salud de la población ${ }^{19-21}$. En los hospitales magnéticos, en los que los entornos son favorables en general para todos los profesionales y en particular para las enfermeras, que pueden poner en práctica sus conocimientos y experiencia clínica, el riesgo de burnout e insatisfacción laboral es menor, hay mejores cuidados y resultados en los pacientes, siendo menor la intención de abandonar la profesión ${ }^{22}$.

Seis años después del inicio de la crisis, los cambios en el entorno sanitario han llevado a unas CT difíciles para las enfermeras, observándose situaciones de malestar, insatisfacción, estrés, burnout y enfermedad. En este contexto nos planteamos explorar qué aspectos mejorarían la CVL desde el punto de vista de las enfermeras.

\section{Metodología}

El presente estudio forma parte de la investigación "Calidad de vida laboral de las enfermeras: evaluación y propuestas de mejora", que tiene como objetivos evaluar la CVL de las enfermeras de la provincia de Barcelona e identificar las acciones de mejora. El diseño fue descriptivo y transversal. La población fueron las enfermeras de la provincia de Barcelona en activo en junio de 2014. El tamaño de la muestra estimada fue de 1744 enfermeras para una población finita de 32463 , con una precisión del $3 \%$, un intervalo de confianza del $99 \%$ y una proporción esperada del $50 \%$. La muestra estuvo compuesta por 1760 enfermeras que estaban en disposición activa para el trabajo entre el 12 de julio de 2014 y el 15 de enero de 2015 . La técnica de muestreo fue no probabilística y consecutiva. La captación de los participantes fue mediante una nota informativa, que fue difundida a través de los canales de comunicación del Colegio Oficial de Enfermeras y Enfermeros de Barcelona, que informaba a las enfermeras sobre la investigación y les daba acceso a un formulario on-line que recogía las respuestas y las almacenaba en la base de datos de la investigación. De las 1792 respuestas recibidas fueron excluidas 32 (16 por duplicación, 3 por no ejercer de enfermeras y 13 por no trabajar en la provincia de Barcelona). El instrumento utilizado en la investigación recogió información sobre datos sociodemográficos y laborales, medidas de contención del gasto, CT, bienestar laboral, 
burnout, engagement, adecuación del tiempo de trabajo, significado del trabajo, impacto positivo y negativo del trabajo y acciones de mejora de la CVL.

El presente estudio da respuesta a uno de los objetivos específicos de la investigación, dando a conocer los aspectos que según la opinión de las enfermeras mejorarían su CVL. Para definir el perfil de la muestra se realizó un análisis descriptivo de las características sociodemográficas y laborales de las participantes a partir de la información obtenida en 12 preguntas de respuesta cerrada, incluidas en el instrumento de investigación; los datos fueron analizados mediante el programa SPSS Statistics 23 y fueron expresados en forma de porcentajes. Para conocer los aspectos que mejorarían la CVL desde la perspectiva de las enfermeras, se realizó un análisis cualitativo de contenido, de tipo exploratorio-descriptivo, a partir de la información obtenida en la pregunta: “¿Qué considera que se podría hacer para mejorar la calidad de vida laboral de las enfermeras?". Esta pregunta, que formaba parte del instrumento de trabajo, tenía un campo abierto donde las participantes respondían sin tener límite de caracteres. La información obtenida se codificó por categorías y éstas se agruparon en áreas temáticas (tabla 1). En el proceso de análisis se realizó una triangulación de investigadores, donde dos de ellos leyeron individualmente las respuestas, codificaron y categorizaron los datos y finalmente los pusieron en común. La información obtenida llegó a la saturación de los datos.

\section{Organización, entorno material y prevención de riesgos}

Entorno físico (instalaciones, equipamientos, recursos materiales y técnicos).

Riesgos laborales.

\section{Organización y entorno s ocial}

Apoyo y respeto profesional en el equipo de trabajo.

Reconocimiento social y laboral.

\section{Organización y método: regulación}

Calidad del contrato laboral.

Conciliación del trabajo con la vida familiar y social.

Tiempo de trabajo (horarios, ritmos, descansos, etc.)

Retribución económica.

Carga de trabajo.

Organización general del trabajo.

Ratio enfermera/paciente.

\section{Organización y método: des arrollo}

Apoyo recibido desde la institución y por el personal directivo.

Reconocimiento laboral (institución y equipo directivo).

Autonomía en la toma de decisiones profesionales.

Participación en las decisiones clínicas y de la organización.

Liderazgo.

Promoción profesional.

Desarrollo y carrera profesional.

Tabla 1: Áreas y Categorías temáticas del análisis cualitativo de contenido.

La investigación fue aprobada para su desarrollo por la Comisión de Bioética de la Universidad de Barcelona. Los aspectos éticos que se tuvieron en cuenta fueron los siguientes: se informó antes de la participa- ción sobre el objetivo de la investigación y el uso científico que se haría de la información; la participación fue voluntaria y se garantizó el anonimato y la confidencialidad de los datos. 


\section{Resultados}

La muestra se caracterizó por ser predominantemente mujeres $(85,9 \%)$, con una edad media de 41,9 años (DE 10,4) y graduadas hacía 18,3 años (DE 10,8). Mayoritariamente participaron enfermeras generalistas $(95,5 \%)$, empleadas en centros de carácter mixto $(57,7 \%)$ y público $(32,7 \%)$, con un contrato estable (56,8\% fijo y $16,2 \%$ funcionario/estatutario), tenían una antigüedad media de 13,5 años (DE 10,0), trabajaban a jornada completa $(76,1 \%)$, habían estado contratadas todo el último año $(86,5 \%)$ y desarrollaban su trabajo en el ámbito asistencial (81\%), en un hospital $(53,3 \%)$ y en un turno diurno $(80,5 \%)$.

Un 99,43\% respondieron la pregunta planteada y opinaron sobre aspectos que mejorarían las CT y la CVL. Una enfermera decía: "No creo que haya una sola medida que pueda mejorar la calidad de vida laboral de las enfermeras, las expectativas son individuales y los puestos de trabajo también" (E-73). A continuación se muestran los aspectos manifestados, aglutinados en cuatro áreas temáticas (tabla 1) que relacionan la organización con: el entorno material y la prevención de riesgos, el entorno social, el método -regulación-y el método-desarrollo-.

En el área de organización, entorno material y prevención de riesgos, opinaron que el entorno físico requería una adecuación de las instalaciones, los equipamientos y los recursos materiales y técnicos, aspectos que mejorarían la organización, los cuidados, la seguridad, el confort, la eficacia, la eficiencia y la calidad. También identificaron situaciones de riesgo laboral como la carga, la sobrecarga y la presión laboral, la tensión y el estrés, el cansancio y el agotamiento, el desgaste, la despersonalización, la desmotivación, la insatisfacción, los abusos de poder, el burnout y el mobbing. Expresaron situaciones de riesgo como: "Ir a trabajar a golpe de teléfono es algo que quema mucho y que hace que vivas un poco sólo por el trabajo" (E456), "No estamos bien tratadas, hacemos de todo para todos, cada día nos ponen más trabajo por lo mismo o por menos y sin recompensa ninguna,..." (E-1645) o “... como un número que te pueden utilizar para ir de arriba a abajo sin tener en cuenta el estrés de tener que asumir servicios que no conoces y eso día a día" (E915). Ante las situaciones de riesgo vividas, propusieron acciones para prevenirlas, corregirlas o minimizarlas, entre ellas, cabe destacar: tener recursos adecuados que facilitaran e hicieran el trabajo más eficiente; disminuir el ratio de pacientes por enfermera; mejorar la comunicación, el respeto, el reconocimiento y el apoyo desde la dirección y los mandos intermedios; valorar periódicamente la satisfacción laboral; disponer de espacios de relax; tener soporte emocional para canalizar las emociones; ubicar a los profesionales de mayor edad o con problemas de salud en puestos de trabajo adecuados; aplicar medidas de conciliación; y avanzar la edad de prejubilación y de jubilación.

En el área de organización y entorno social, refirieron que eran "profesionales formados y muy valiosos para la atención y el desarrollo habitual en cualquier ámbito sanitario" (E- 153), a las que se debería reconocer las "competencias y valor profesional" (E-154) que aportaban, "la responsabilidad" (E-802) que asumían y "el esfuerzo y el trabajo realizado día a día" (E-38). Sin embargo, percibían una falta de apoyo, valoración, respeto y reconocimiento de los compañeros, fundamentalmente médicos, pero también de la institución y del personal directivo como veremos más adelante. En relación a los compañeros opinaron que deberían reconocer el trabajo que desempeñan dentro del equipo. También afloró la percepción de falta de reconocimiento social de los políticos, de los ciudadanos, de los usuarios y de los familiares, considerando que era necesario que se diera más visibilidad y reconocimiento social a su trabajo. Una enfermera proponía: "Enseñar a la población que también existimos y no somos apéndices del médico"(E-1637).

En el área de organización y método -regulación-, opinaron respecto a la calidad del contrato laboral valorando que había precariedad laboral, determinada en parte por la temporalidad y la corta duración de los contratos. Una enfermera verbalizaba: "La calidad en la contratación en nuestro sector es pésima, con contratos temporales, en muchos casos de días, incluso horas sueltas, conllevando una gran incertidumbre y dificultad para sobrellevar una vida normal al no poder planificar tus actividades por tener que estar pendiente siempre de una llamada telefónica" (E-1043). Situaciones como esta les generaba incertidumbre, inestabilidad (por la falta de continuidad laboral y por los cambios de ámbito y de turno), dificultad para la conciliación e inseguridad, que una enfermera refería diciendo: "No sabes si trabajarás un mínimo para poder pagar los gastos" (E-456). Opinaron que si la contratación tuviera continuidad y estabilidad mejoraría su seguridad, su conciliación y su CVL. También propusieron para mejorar la conciliación "horarios más estables" (E502), "turnos que permitieran mayor conciliación" (E919), "facilitar la flexibilidad horaria" (E-197), "más 
fines de semana y festivos con la familia" (E-632), "más días de libre disposición” (E-383) y "mejores contratos" (E-1059).

En relación al tiempo de trabajo, propusieron disminuir la jornada laboral anual y aumentar los días de fiesta hasta recuperar las condiciones previas a la crisis.

Las enfermeras opinaron que la retribución económica había de ser digna, justa y acorde al nivel de formación, especialización, competencias y responsabilidades. También había de reconocer el trabajo desarrollado, las cargas asumidas, los riesgos existentes y la productividad. Sin embargo muchas participantes expresaron desacuerdo con la retribución y pérdidas retributivas durante la crisis que expresaban diciendo: "Cada vez tenemos más trabajo, lo tenemos que realizar en peores condiciones y encima estamos cobrando menos..." (E-1722). Algunas de las propuestas planteadas fueron la recuperación de las pérdidas y la equiparación retributiva entre centros, con profesionales con titulaciones del mismo nivel y con enfermeras europeas.

La presión, la carga, la sobrecarga y el desajuste entre tiempo y actividad, son términos que los relacionaron con el bajo ratio enfermera/paciente pero también con una organización inadecuada del trabajo. La presión asistencial la asociaron al cierre de camas hospitalarias, al déficit de personal, a la mayor complejidad de pacientes y a las demandas o exigencias de pacientes, familiares, médicos y dirección. La carga y la sobrecarga la relacionaron con la mayor complejidad y dependencia de los pacientes, el déficit de personal, la ausencia o no idoneidad de los recursos materiales disponibles y la organización inadecuada del trabajo, aspectos que una enfermera expresaba diciendo: "En estos momentos se está trabajando con mucha carga asistencial provocada por los recortes, la comorbilidad y el envejecimiento de la población y esto cada vez irá a más. Se debería intentar repartir las cargas asistenciales..." (E-1143). El tiempo disponible y la actividad a desarrollar lo asociaron a la necesidad de hacer el trabajo pudiendo atender, escuchar, visitar, acompañar y dar cuidados integrales a los pacientes, así como realizar actividades no asistenciales vinculadas a la profesión. Para mejorar estas situaciones propusieron analizar y redistribuir las cargas, medir los tiempos de ejecución de las tareas, así como adecuar el ratio enfermera/paciente a la actividad, al tiempo necesario para desarrollarla, a la complejidad de los pacientes y a la carga asistencial.
“Tener más tiempo para atender los pacientes y no tener una visita cada 10 minutos o una sola enfermera llevar 15-20 pacientes a la vez" (E-835) es lo que mejoraría la CVL para esta enfermera y para otras que asociaron la CVL con el ratio enfermera/paciente considerando que mejoraría si se dejara de recortar personal, se sustituyeran las ausencias y se aumentara el personal. Opinaron que mejorar el ratio les permitiría disminuir la carga y la presión asistencial, desarrollar el rol autónomo y de colaboración, dedicar más tiempo a cada paciente, dar una atención y cuidados personalizados, mejorar la seguridad y la calidad asistencial, trabajar con menos estrés y prevenir el desgaste personal.

En el área de organización y método -desarrollo-, manifestaron falta de apoyo, respeto, valoración y reconocimiento desde la institución, la gerencia, la dirección y los mandos intermedios. Una enfermera afirmaba: “... se debe valorar el trabajo que desempeñamos tanto por parte del usuario como de la institución. Eres un número y tu situación personal no cuenta. No se valora los sacrificios que puedas hacer por la empresa" (E-1191) y otra decía que le gustaría "sentirse más valorada por la organización y que se promueva tu participación e implicación en el servicio"(E-1371). En general opinaron que sería necesario que: las organizaciones valoraran más su trabajo y las trataran mejor; la gerencia apoyara el proyecto y el liderazgo de las enfermeras; la dirección las escuchara, valorara, diera respuesta a los problemas, las hiciera participar en las decisiones, las apoyara, las motivara y fomentara su desarrollo profesional; y los mandos intermedios las escucharan, las ayudaran y las apoyaran. Sentirse apoyadas, valoradas, respetadas y reconocidas mejoraría su CVL.

Una enfermera demandaba "mayor autonomía en la toma de decisiones en relación a la organización del trabajo y la asistencia..." (E-1160) y en general opinaron que era necesario aumentar la autonomía en el desarrollo de su propio rol y competencias, pero también en la gestión, la organización, la distribución del trabajo y la toma de decisiones. Propusieron empoderar a las enfermeras y recuperar el liderazgo perdido, su capacidad de organización y de gestión de los cuidados.

En la participación de las enfermeras en las decisiones organizativas y clínicas, consideraron que los líderes enfermeros habían de participar en los órganos de decisión y las enfermeras en las decisiones asistenciales y en la propia organización, planificación y método 
de trabajo. Pidieron que la dirección las informara, las hiciera partícipes de los proyectos y tuviera en cuenta su opinión antes de aplicar cambios. Una enfermera decía: "Que seamos escuchadas por las personas que nos pueden facilitar las mejoras en el trabajo y que nos dejen hacer los cuidados más personalizados y según las necesidades de cada paciente" (E-448).

En relación al liderazgo, opinaron que la organización, la gestión, la planificación, la participación, la implicación y la toma de decisiones de las enfermeras habían de mejorarse. Consideraban que las organizaciones habrían de estar menos burocratizadas y estar lideradas por personal sanitario competente, comprometido y con experiencia asistencial, debiéndose revisar los cargos de gestión periódicamente y valorar su continuidad o sustitución. Las direcciones enfermeras habían de tener liderazgo y proyecto enfermero y en los organigramas tenían que estar al mismo nivel y con el mismo poder de decisión que los médicos. Las enfermeras gestoras habían de ser líderes profesionalizadas y competentes, formadas y con conocimiento de la profesión, tener habilidades comunicativas y actitudes idóneas, estar implicadas en el proyecto, ejercer un liderazgo personalizado y de buen trato, así como tener poder de decisión. Una enfermera afirmaba: "Es necesario un liderazgo de la dirección enfermera, con un proyecto respetado y apoyado por la dirección general y otras categorías profesionales, liderado por los mandos intermedios y en el que las enfermeras se impliquen y participen activamente" (E-1).

Las participantes opinaron de la promoción profesional que habría de: mejorarse las oportunidades y la accesibilidad; ser objetiva, equitativa y justa; valorar las competencias, la antigüedad y "facilitar la promoción dentro del servicio en función de las capacidades y la formación del profesional" (E-737); dar oportunidades de mejora laboral y de desarrollo profesional. En relación al desarrollo profesional, opinaron que se habría de: definir una carrera profesional única para todo el colectivo; introducir, mejorar o valorar más la carrera profesional y ayudar a los profesionales a poder avanzar en ella; regular distintas categorías en relación a los niveles de carrera profesional; introducir en los niveles elementos de cambio e incentivación y actividades o competencias de mayor responsabilidad; e incluir la formación, docencia e investigación dentro de la jornada laboral.

\section{Discusión}

Muchos de los resultados de este estudio, como veremos a continuación, están presentes en otras investigaciones. Los déficits identificados en las CT, relacionados con la carga, la autonomía, la participación, las condiciones de empleo y el desarrollo profesional, entre otros aspectos, el Instituto Nacional de Seguridad e Higiene en el Trabajo (INSHT) ${ }^{8}$ y diferentes auto$\operatorname{res}^{5,9-14}$ los han considerado factores de riesgo laboral que pueden llevar a situaciones de malestar, insatisfacción, estrés, burnout y enfermedad. Aspectos como el desacuerdo con la retribución, la promoción, el liderazgo, la organización del trabajo, los compañeros de trabajo y la comunicación, autores como Panagiotis ${ }^{23} \mathrm{y}$ Masum $^{24}$ los han asociado a insatisfacción. Otros autores han considerado como causas de malestar, estrés e insatisfacción situaciones como la falta de personal, el poco reconocimiento entre médicos y enfermeras ${ }^{17,25}$, la falta de reconocimiento de la institución y los conflictos en el lugar de trabajo ${ }^{26}$, la falta de soporte del equipo de trabajo, los contratos laborales irregulares, el aumento de las cargas asistenciales, la fatiga psicológica, ${ }^{5,11-14}$, la precariedad en la contratación, la insegu$\operatorname{ridad}^{11,12,14}$, la excesiva exigencia emocional y el desequilibrio entre la vida profesional, personal y fami$\operatorname{liar}^{10,27}$. Autores como Aiken han afirmado que pueden generar estrés crónico, burnout, insatisfacción profesional y abandono de la profesión ${ }^{15}$. Las CT asociadas a conflictos interpersonales, las dificultades en las relaciones entre médicos y enfermeras, la falta de autonomía y autoridad en la toma de decisiones y la indefinición del papel profesional.

También hay estudios que han evidenciado los efectos beneficiosos que generan unas CT óptimas y que se corresponden con algunas de las acciones de mejora propuestas por las participantes. Autores como Lashinger $^{28}$ han mostrado que el ejercicio de un buen liderazgo, realizado desde la transparencia, con buena comunicación, con soporte a los profesionales y con recursos para el trabajo y para la formación, conlleva un mayor compromiso de los trabajadores y lleva a una mejor relación en los equipos, aumentando la satisfacción laboral y la productividad. Otros estudios han mostrado que las enfermeras que aplican sus habilidades en el trabajo, desarrollándolo autónomamente y evaluándolo, tienen mayor empoderamiento y una actitud más positiva respecto al trabajo, mayor satisfacción laboral y presentan menos ansiedad ${ }^{29}$. Autores como KutneyLee $^{22}$ han afirmado que en los hospitales magnéticos hay mejores cuidados y resultados en los pacientes. 


\section{Conclusiones}

La percepción de las enfermeras es que su CVL aumentaría si mejoraran las CT relacionadas con el entorno físico y material, la prevención de riesgos laborales, el reconocimiento, la contratación, la conciliación, el tiempo de trabajo, la retribución, la carga y la organización del trabajo, el ratio enfermera/paciente, la autonomía, la participación, el liderazgo, el desarrollo y la promoción profesional. Los resultados obtenidos permiten concluir que las expectativas de mejora de la CVL son individuales y parecen estar asociadas a condiciones laborales, profesionales y personales de cada enfermera.

Es necesario que las organizaciones implementen acciones dirigidas a mejorar las CT y a conseguir un entorno laboral confortable, que como ya se ha evidenciado en diferentes estudios3,15 además de repercutir positivamente en el bienestar y la satisfacción de las enfermeras, también conllevará una mejora en la calidad de la atención, en la seguridad y la satisfacción de los pacientes, así como en los resultados de salud de la población.

\section{Bibliografía}

1. Blanch JM, Sahagún M, Cervantes G. Estructura factorial del cuestionario de condiciones de trabajo. Revista de Psicología del Trabajo y de las Organizaciones. 2010;26 (3): 175-189.

2. Ley $31 / 1995$, de 8 de noviembre, de Prevención de Riesgos Laborales. Boletín Oficial del Estado, $n^{\circ} 269$, (10 de noviembre de 1995). [citado 4Sep 2016]. Disponible en:

https://www.boe.es/boe/dias/1995/11/10/pdfs/A3259032611.pdf

3. Aiken LH, Sermeus W, Van den Heede K, Sloane DM, Busse R, McKee $M$, et al Patient safety, satisfaction, and quality of hospital care: cross sectional surveys of nurses and patients in 12 countries in Europe and the United States. BMJ. 2012; 344: e1717. doi: http://dx.doi.org/10.1136/bmj.e1717 [citado 8 May 2016]. Disponible en: http://www.bmj.com/content/bmj/344/bmj.e1717.full.pdf.

4. Ochoa P, Blanch JM. Work, malaise, and well-being in Spanish and Latin-American doctors. Rev Saúde Pública 2016; 50: 21. Doi: 10.1590/S1518-8787.201605000600 [citado 13 sep 2016] Disponible en: http://www.scielo.br/pdf/rsp/v50/0034-8910-rspS1518-87872016050005600.pdf

5. Lavoie-Tremblay M, Bonin JP, Lesage A, Bonneville-Roussy A, Lavigne $G$, Laroche $D$. Contribution of the psychosocial work environment to psylchological distress among health care professionals before and during a major organizational change. The health care $\mathrm{m}$ a n a ge r $2010 ; 20(4): 293-304$. do i : 10.1097/HCM.0b013e3181fa022e.

6. Mansilla F. Manual de riesgos psicosociales en el trabajo. Teoría y práctica. [Monografía en Internet]. Valencia: Psicología on line. Formación, autoayuda y consejo on line. [citado 13 sep 2016]. Disponible en: http://www.psicologia-online.com/ebooks/riesgos/

7. Sánchez- Anguita A. Salud Laboral. Autoeficacia, ansiedad y satisfacción. Salamanca: Amaru Ediciones; 2006.
8. Cuenca R. Introducción a la prevención de riesgos laborales de origen psicosocial. Documento divulgativo. INSHT. Madrid: Ministerio de Empleo y Seguridad Social; 1996.

9. Jourdain G, Chênevert D. Job-demands-resources, burnout and intention to leave the nursing profession: A questionnaire survey. Int J Nurs Stud. 2010;47(6): 709-22. doi: 10.1016/j.ijnurstu.2009.11.007. PubMed PMID: 20138278

10. Cortese C, Colombo L, Ghislieri C. Determinants of nurses' job satisfaction: the role of work-family conflict, job demand, emotional charge and social support. J Nurs Manag 2010; 18(1): 35-43. oi: 10.1111/j.1365-2834.2009.01064.x. PubMed PMID: 20465727.

11. Appelbaum D, Fowler S, Fiedler N, Osinubi O, Robson M. The impact of environmental factors on nursing stress, job satisfaction and turnover intention. J Nurs Adm. 2010; 40 (7-8): 323-8. doi: 10.1097/NNA.0b013e3181e9393b. PubMed PMID: 20661062.

12. Riahi S. Role stress amongst nurses at the workplace; concept analysis. J Nurs Manag. 2011; 19(6): 721-31. doi: 10.1111/j.1365-2834.2011.01235.x. Epub 2011 Aug 10. PubMed PMID: 21899625

13. Arterman A, Colom J, Gabilondo A, Massachs E. La salut i el benestar dels professionals sanitaris en l'entorn laboral. Guia de bones pràctiques per a institucions, equips i professionals. [Monografia en internet] Barcelona: Generalitat de Catalunya. Agència de Salut pública de Catalunya; 2012. [Citado el 13 sep 2016] disponible en:

http://www20.gencat.cat/docs/canalsalut/Minisite/Drogues/D estacats/Arxius/Guia\%20Salut\%20i\%20benestar.pdf

14. Purcell S, Kutash M, Cobb S. The relationship between nurses' stress and nurse staffing factors in a hospital setting. J Nurs Manag. 2011. 19(6): 714-20. doi: 10.1111/j.13652834.2011.01262.x. PubMed PMID:21899624.

15. Aiken LH, Clarke SP, Sloane DM, Sochalski J, Silber JH. Hospital nurse staffing and patient mortality, nurse burnout, and job dissatisfaction. JAMA. 2002; 288(16): 1987-93.

16. Villalobos GH. Vigilancia Epidemiológica de los Factores Psicosociales. Aproximación Conceptual y Valorativa. Ciencia y Trabajo.[Internet] 2004 Oct-Dic [Citado 13 sep 2016]: 6(14): 197-201 [aprox. 4p.]. Disponible en:

http://www.huila.gov.co/documentos/V/vigilancia_epidem_psi cosociales.pdf

17. Pipe TB, Buchda V, Launder S, Hudak B, Hulvey L, Karns K et. al. Building personal and professional resources of resilience and agility in the healthcare workplace. Stress Health. 2012; 28: 1122. doi: 10.1002/smi.1396. PubMed PMID: 22259154.

18. Letvak S, Ruhm C, Lane S. The impact of nurses' heath on productivity and quality of care. J Nur Adm. 2011; 41(4): 162-7. doi: 10.1097/NNA.0b013e3182118516. PubMed PMID: 21430464

19. Pérez-Ciordia I, Guillén-Grima F, Burgos A, Aguinaga I. Satisfacción laboral y factores de mejora en profesionales de atención primaria. Anales Sis San Navarra [Internet]. 2013 May-Ago [Citado 13 sep 2016]; 36(2): 253-62 [aprox. 9p.]. Disponible en: http://scielo.isciii.es/pdf/asisna/v36n2/original6.pdf

20. Bullich-Marin I, Miralles-Basseda R, Torres-Egea P, PlanasCampmany C, Juve-Udina ME. Evaluación del ambiente de trabajo de las enfermeras en las unidades sociosanitarias de atención intermedia de Cataluña. Rev. Esp. Gerontol. Jun 2016; 51(3). doi: 10.1016/j.regg.2015.10.009. Disponible en: http://dx.doi.org/10.1016/j.regg.2015.10.009

21. Kilánska D, Gorzkowicz B, Sienkiewicz Z, Lewandowska M, Dominiak I, Bielecki W. Evaluation of chosen determinants of the positive practice environments (PPE) at polish nursing wards. Med Pr 2016;67(1):11-9. doi: 10.13075/mp.5893.00225. PubMed PMID: 27044715.

22. Kutney-Lee A, Solane DM, Cimiotti JP, Quin LW, Aiken LH. Changes in Patient and Nurse outcomes associated with magnet hospital recognition. Med Care. Jun 2015;53(6)550-557. doi: 10.1097/MRL.0000000000000355. PubMed PMID: 25906016. Pub Med Central PMCID: PMC4431919. 
23. Panagiotis I, Katsilakavili V, Galanis P, Velonakis E, Papadatoi D, Sourtzi P. Impact of Job satisfaction on Greek nurses' Healthrelated Quality of life. Saf Health Work. [Internet] 2015 Dec [Citado 13 sep 2016];6(4):324-8 àpprox. 4 pa.]. doi: $10.1016 / \mathrm{j}$.shaw.2015.07.010. Disponible en: http://www.sciencedirect.com/science/article/pii/S209379111 5000669

24. Masum AKM, Azad MAK, Hoque KE, Beh L-S, Wanke P, Arslan Ö. Job satisfaction and intention to quit: an empirical analysis of nurses in Turkey. PeerJ. 2016 Abr 4:e1896. Doi:10.7717/peerj.1896. PubMed PMID: 27168960

25. Papathanassoglou E, Karanikola M, Kalafati M, Giannakipoulou M, Lemonidou C, Albarran J. Professional autonomy, collaborations with physicians, and moral distress among European intensive care nurses. Am J Crit Care. 2012 Mar; 21(2): 41-52. doi: 10.4037/ajcc2012205.Pub Med PMID: 22381995.

26. Faler, M, Gates M. Work-related Burnout, job satisfaction, intent to leave, and nurse-assessed quality of care among travel nurses. $J$ Nur Adm. 2011 fe b; 4 1 (2): $71-7$. do i : 10.1097/NNA.0b013e3182059492. PubMed PMDI: 21266885.

27. Larrabee J, Wu Y, Persily C, Simoni P, Johnson P, Marcischak T, et alt. Influence of stress resiliency on RN job satisfaction and intent to stay. West J Nurs Res. 2010; 32(1): 81-102. doi: 10.1177/0193945909343293. Pub Med PMDI: 19915204.

28. Laschinger $\mathrm{H}$, Wong $\mathrm{CA}$, Grau AL. Authentic leadership, empowerment and burnout: comparison in new graduates and experienced nurses. J Nurs Manag. 2013 Apr;21(3):541-52. doi: 10.1111/j.1365-2834.2012.01375.x. PubMed PMID: 23405976.

29. Kuokkanen L, Leino-Kilpi H, Numminen O, Isoaho H, Flinkman M, Meretoja R. Newly graduated nurses' empowerment regarding profesional competence and other work-related factors. BMC Nursing. 2016; 15-22. Doi: 10.1186/s12912-016-0143-9. PubMed PMID: 27013927. PubMed Central PMCID: PMC4806465. 